\title{
ÁMBITO DE LA PEDAGOGÍA SOCIAL EN AMÉRICA LATINA
}

\author{
NELSON TORRES VEGA \\ Facultad de Educación, \\ Universidad de Nariño.
}

\section{RESUMEN}

El ámbito socio-pedagógico es el espacio disciplinar por excelencia, desde el cual se plantea la praxis de la Educación Social que propicia acciones cultivadas con los sujetos en condiciones de marginalidad e incluye su dimensión social. Es esta dimensión la que les aporta identidad como individuos, es la perspectiva sociológica de la interrelación que se realiza con individuos que se define mediante el concepto de acción social, entendida como"conducta orientada intencionalmente, de los diversos agentes sociales que constituyen un sistema de interrelaciones" (Pérez, G. 2003, p. 143). Los destinatarios de la acción socioeducativa son sujetos, grupos colectivos específicos y sectores de población de diversas características, ya sea por su edad, por su género, por su situación laboral, por su nivel educativo y cultural; por sus dificultades de socialización (física, psíquica, sociales, culturales), por su origen y ubicación en el territorio; en síntesis y de acuerdo a las problemáticas de las personas, se puede mencionar a la población en riesgo social (delincuencia, marginación, desplazamiento, exclusión, dependencia); y finalmente población en general (atención del adulto, tercera edad, desarrollo local, animación sociocultural).

El presente texto intenta describir los diversos espacios de aplicación de la Pedagogía Social en América Latina, concepto que ha estado muy cercano a la denominada educación popular, educación de adultos o educación para poblaciones en condición de vulnerabilidad, marginalidad y pobreza. Se pretende instaurar propuestas para la transformación social y educativa de la región latinoamericana, mediante un modelo de sociedad igualitaria sustentada en la formación de un nuevo ciudadano.

Palabras clave: Pedagogía Social, Población Vulnerable, Ámbitos Socioeducativos, Transformación Social.

\section{ABSTRACT}

The socio-pedagogical field is the disciplinary space par excellence, from which the praxis of Social Education is proposed which favors actions cultivated with the subjects in conditions of marginality and includes their social dimension. It is this dimension that gives them identity as individuals, it is the sociological perspective of the interrelation that is realized with individuals that is defined by the concept of social action, under- 
stood as"intentionally oriented behavior, of the diverse social agents that constitute a System of interrelations"(Pérez, G. 2003 p.143). The beneficiaries of socio-educational action are subjects, specific groups and sectors of population of different characteristics, whether by age, gender, employment status, educational or cultural level; By their difficulties of socialization (physical, psychic, social, cultural), by their origin and location in the territory; In synthesis and according to the problematic of the people, it is possible to mention the population at social risk (delinquency, marginalization, displacement, exclusion, dependence); And finally population in general (adult care, senior citizens, local development, socio-cultural animation.

The present text tries to describe the diverse spaces of application of Social Pedagogy in Latin America, a concept that has been very close to the so-called popular education, adult education or education for vulnerable populations, marginality and poverty. It intends to establish proposals for the social and educational transformation of the Latin American region, through a model of egalitarian society based on the formation of a new citizen.

Key words: Social Pedagogy, Vulnerable Population, Socio-educational Spheres, Social Transformation.

\section{Introducción}

El devenir de la Pedagogía Social en América Latina ha estado muy cercana a los desarrollos conceptuales de la denominada educación popular, educación de adultos, educación para poblaciones en condición de vulnerabilidad, desplazamiento forzado, marginalidad y pobreza; con sus diferencias y matices, pero con un eje articulador común: repensar la educación y diseñar propuestas de cambio para la transformación social. Su característica ha sido instaurar en la región un modelo de sociedad igualitaria, contribuir con la formación de un nuevo ciudadano, en respuesta al modelo neoliberal hegemónico e impuesto que ha tenido como resultado el aumento de las desigualdades. Es decir, así como en Europa (Alemania y España especialmente) se configuró una disciplina, con saberes y prácticas educativas para apoyar a los "necesitados", Latinoamérica y Colombia construyen, desde hace varias décadas, una heterogeneidad de aprendiza- jes, prácticas sociales y populares, tanto en comunidades rurales como urbanas, donde la institución escolar ha sido uno de los ejes de estos desarrollos, que le han venido dando legitimidad a la Pedagogía Social, pero sin los resultados esperados.

Estas características compartidas entre la Educación Social y la Educación Popular en América Latina conducen a plantear algunos desafíos propios para esta región que aboga permanentemente por su liberación cultural, económica, ideológica y política (Arias, $\mathrm{R}$. L. 2006; Krichesky, M. 2011; Monteiro, E. 2011; Nájera, E. 2011); estos desafíos son, entre otros:

Estudiar y atender las necesidades educativas y sociales dentro y fuera de la escuela, a todos los grupos etarios, demandas relacionadas con la diversidad cultural, poblaciones en riesgo: pobreza extrema, desplazamiento forzado, entre otras. 
Desarrollo de acciones para prevenir y perseguir los crímenes de lesa humanidad, contra el patrimonio cultural, la ecología y en general aquellos delitos que violen los derechos humanos.

Apoyar, desde los sistemas educativos, a los países de la región en la lucha por una mayor equidad social y educativa, especialmente de niños, niñas y jóvenes de los sectores más vulnerables que no se han podido educar.

Constituirse en plataforma de pensamiento e interacción socioeducativa con enfoques teórico- prácticos propios, que respondan al contexto socio-histórico latinoamericano, generando movimientos sociales contra-hegemónicos y alternativos, respetando la pluralidad de actores e ideas, siempre apoyados en tendencias pedagógico-políticas articuladas para la defensa de la educación pública y de calidad.

Propiciar el análisis crítico de las políticas sociales y de los modelos socioeducativos que se insertan en la región como dispositivos sociales de poder, a la vez contribuir en el diseño de nuevos modelos de acción social y educativa, mediante la convocatoria a los educadores y a quienes se están formando a participar activamente, como sujetos capaces de reflexión desde y sobre sus experiencias.

Es evidente que estos desafíos, y otros no enunciados, para la Pedagogía Social en América Latina, se inspiran inicialmente en el enfoque crítico-social de la Escuela de Frankfurt, posteriormente complementado con los aportes teóricos de autores como Paulo Freire (1976; 1982), Orlando Fals Borda (1989), Henry Giruox (1990),
Peter MacLaren (2003), Carr y Kemmis (1993), Jhon Elliot (1986), Donald Schon (2010) entre otros. De esta manera las prácticas educativas actuales sirven de nicho para que la Pedagogía Social en Colombia tenga identidad propia, creando sus líneas de estudio, formación e investigación.

Diferentes autores como Fermoso, P. (1991), Pérez, G. (2009), Sarrate y Sanz (2009), Sáez. J. (1994), entre otros, están de acuerdo en que la Pedagogía Social es parte de la Pedagogía General y ésta a la vez parte de las Ciencias Humanas y Sociales o también llamadas por Dilthey, W. (1833-1911) como Ciencias del Espíritu ${ }^{1}$. Es en esta perspectiva que se hace alusión, por una parte a los ámbitos de la Pedagogía Social, como la socialización, la ayuda vital y la ayuda social; por otra parte se distinguen áreas o escenarios de atención desde la concepción de "pedagogía de la necesidad" o ciencia práctica social y educativa, que inicialmente fue pensada para intervenir fuera de la escuela y la familia, es decir como educación no formal; sin embargo, la crisis creciente presentada en estos espacios, ha obligado a que la Pedagogía Social se extienda hacia los ámbitos formales y reglados de la educación.

Las denominadas "ciencias del espíritu" son aquellas disciplinas que se ocupan del estudio de la cultura, por tanto su fundamento epistemológico es diferente al de las ciencias naturales;

1. Las ciencias del espíritu es la denominación dada por Dilthey a las Ciencias Humanas al referirse a un concepto epistemológico "que designa a un grupo de ciencias cuyo objeto es el hombre o los grupos humanos y su cultura". 
éstas son explicativas y de corte positivista, aquéllas buscan la "comprensión (Verstehen) de los hechos y expresiones culturales del ser humano y esta comprensión es posible en tanto el objeto de estudio no es lo externo al sujeto, sino que hace parte de sus realidades espirituales y culturales (Briones, G. 2002). En consecuencia el campo de la Pedagogía Social es el ser humano en sus diferentes dimensiones, especialmente sus necesidades, sus angustias, sus carencias, su devenir como ser social.

\section{Posibles Ámbitos de la Pedagogía Social}

Para ser coherentes con lo dicho y teniendo como premisa la concepción de la pedagogía/ educación social como disciplina flexible, abierta y dinámica, que desarrolla su acción en escenarios, algunos previsibles, otros inciertos, pero siempre a la par de los cambios sociales de momento, se debe partir de una formación de profesionales creativos e innovadores, con suficientes herramientas teórico-prácticas para ofrecer una atención educativa diferenciada, especialmente a los niños, niñas y jóvenes en condición de desplazamiento y vulnerabilidad.

García Míguez, J. (2010) presenta una taxonomía amplia de los escenarios donde la pedagogía/ educación social precisa su interacción, teniendo como criterio básico los agrupamientos humanos o modelos centrados en los colecti$\operatorname{vos}^{2}$, entre los que se mencionan: escenarios relacionados con personas mayores;

2. Esta clasificación no es la única. Para el autor García Mínguez, es una de las más completas, donde la aplicación de la Pedagogía Social es más incluyente. población adulta y desempleada; el ocio y el tiempo libre para adultos; colectivos con necesidades especiales; segmento poblacional de la mujer; población de la juventud; sector de la población infantil como son los niños y niñas escolarizados y desescolarizados. Estos escenarios se complementan a continuación, indicando los usuarios en cada caso:

1. Escenarios relacionados con personas mayores: personas adultas mayores, personas mayores con autonomía, personas adultas y mayores autónomos, personas mayores autónomos y dependientes.

2. Sector de la población adulta y desempleada: Adultos en general, adultos con diferentes necesidades de formación o promoción o atención.

3. El ocio y el tiempo libre para los adultos: Todo tipo de colectivos adultos: colectivos especialmente jóvenes y familias. Colectivos de adultos en general.

4. Colectivos con necesidades especiales: Adictos a las drogas, excluidos en barrios periféricos o conflictivos, poblados, transeúntes, desplazados, semáforos, personas con diferentes necesidades de formación y ocupación, jóvenes y adultos con necesidades educativas, sociales, de normalización, persona con carencias familiares y problemas de riesgo social, inmigrantes.

5. Segmento poblacional de la mujer: Mujeres en general, Mujeres maltratadas, Mujeres con necesidades específicas, Mujeres con cargas familiares, Mujeres sin titulación alguna, Mujeres con dificultad de inserción social, Madres comunitarias. 
6. Población de la juventud: Jóvenes en general, jóvenes desempleados, jóvenes con problemas de integración y/o justicia.

7. Sector de la población infantil: Niños en general, niños escolarizados.

Como se observa la Pedagogía/Educación Social posee amplios contextos de intervención donde su propósito final es la formación integral de todos los miembros de una sociedad, capaz de garantizar el desarrollo de capacidades y actitudes para pensar, decidir y actuar con autonomía y responsabilidad para lograr la transformación de las realidades cotidianas, como bien lo afirma Arias, R.L. (2006): "La Educación Social potencia la transformación cultural, social, económica, política; a partir de ella es posible incidir en los imaginarios colectivos y las prácticas que comprometen conductas proactivas, conductas de riesgo, situaciones de crisis, dificultades circunstanciales" (p. 119).

\section{La Pedagogía Social en América Latina}

América Latina tiene una larga y rica tradición en aspectos tanto teóricos como prácticos en pedagogía social y la construcción epistemológica corresponde a elementos propios de su realidad, que ha sido enriquecida conceptualmente por los aportes de corrientes europeas, pero sus desarrollos han sido autónomos, propios. La educación social en América Latina es un fenómeno relativamente reciente ${ }^{3}$, que a su llegada

3. Según Triana, Alba Nidia (2011) el tema de la educación social en América Latina "aproximadamente data de veinte años atrás" la misma autora agrega que el origen y conceptualización inicial corresponde a enfoques teóricos y conceptuales de las ciencias sociales, de discursos originados tanto en Europa como en América". de Europa, encuentra en esta región un ambiente predispuesto para ampliar conocimientos y experimentar alternativas metodológicas nuevas de intervenciónacción sobre diversas situaciones como la pobreza, la exclusión social, la violencia, el desplazamiento, entre otros.

\section{Algunos Aportes Teóricos al Desarrollo de la Pedagogía Social Latinoamericana}

Según lo describe Triana, A. (2011) los desarrollos prácticos y experiencias sobre pedagogía social en América Latina, desde mediados de los años 60, están referidas a las propuestas reconocidas como: la educación popular de Paulo Freire (1921-1997), la investigación acción participativa de Orlando Fals Borda (1925-2008), el desarrollo a escala humana de Manfred Max Neef (1932), los aportes del Centro Latinoamericano del Trabajo Social, CELATS. Éstos y otros referentes se constituyen en objeto de debate sobre procesos socioeducativos que según Úcar, X. (2011) la función básica de la pedagogía social que llega de Europa es la de "tratar de conjugar su voz con las voces latinoamericanas" (p. 5) ${ }^{4}$.

Los aportes más importantes de Max-Neef (1986) en el contexto de la pedagogía social para América Latina,

4. El autor ÚCAR $(2011$, p. 5) se refiere a dos elementos claves que marcan la evolución y desarrollo de la pedagogía social en América Latina: a) "La idiosincrasia del continente, que es expresada por los autores de diferentes formas: se habla de la gran diversidad cultural... de la necesidad de pensar la educación desde la hibridez cultural, superando racionalidades instrumentales". b) "El necesario diálogo de la pedagogía social y la educación social, en tanto que teorías, prácticas y experiencias procedentes de otros contextos, con las ideas de Freire y la educación popular que son originarias del continente latinoamericano". 
se sintetizan en la obra "El Desarrollo a Escala Humana" que se sustenta en la satisfacción de las necesidades humanas fundamentales, en propiciar la autodependencia progresiva, de articular al ser humano con la naturaleza y la tecnología, de relacionar los procesos globales con lo local, de lo personal con lo social, de la planificación con la autonomía y de la sociedad con el Estado; "necesidades humanas, autodependencia y articulaciones orgánicas, son los pilares fundamentales que sustentan el Desarrollo a Escala Humana" (MaxNeef, M. 1986, p. 14). Para que estos propósitos sean reales se deben apoyar en el protagonismo de las personas privilegiando la diversidad y la autonomía, logrando que la persona sea sujeto y no objeto de desarrollo, facilitando prácticas democráticas más directas $\mathrm{y}$ participativas, como bien lo describe este autor cuando afirma:

Al facilitar una práctica democrática más directa y participativa puede contribuir a revertir el rol tradicionalmente semi-paternalista del Estado latinoamericano, en rol estimulador de soluciones creativas que emanen desde abajo hacia arriba y resulten, por lo tanto, más congruentes con las aspiraciones reales de las personas (Max-Neef. 1986, p. 15).

Desde Latinoamérica, el pensamiento de Paulo Freire se constituye en un referente y en una tendencia mundial en educación; a través de su reconocida obra pedagógica aportó de manera integral al estudio de la problemática educativa, partiendo de una perspectiva que rompe con los esquemas tradicionales de la pedagogía, centrando su trabajo en los colectivos oprimidos, marginados, desempleados, analfabe- tas, pobres, etc., logrando con ellos y desde ellos una interacción dialógica y emancipadora, generando procesos de liberación de sus condiciones de opresión y desigualdad. Sus obras más importantes fueron, Pedagogía del oprimido, Educación como Práctica de la Libertad y Extensión o Comunicación, además de otra veintena de libros y escritos $^{5}$.

Los aportes de Paulo Freire están inscritos en el contexto de los procesos de desarrollo de la Educación Popular en América Latina, entendida ésta como "un proceso de formación y capacitación dentro de una perspectiva política, forma parte de la acción organizada del pueblo en orden a lograr el objetivo de construcción de una sociedad" (Barreras, A.C. 2013). La educación popular es una categoría con características propias para América Latina, referidas a la educación organizada y destinada a los sectores poblacionales marginados, como campesinos, trabajadores, desempleados, emigrantes, desplazados, mujeres, niños, niñas y jóvenes; por otra parte el fomento y desarrollo de la educación popular ha estado a cargo de las propias organizaciones populares, del sector de los intelectuales, políticos, sociales y educativos, dentro de una lógica emergente.

5. Las obras de Paulo Freire son numerosas. Entre las publicadas están: Sua visão de mundo, de homem e de sociedade (2001); Pedagogia da Autonomia: saberes necessários à prática educativa (1996); Por uma pedagogia da pergunta (1985); Pedagogia do oprimido (1987); Pedagogia da indignação: cartas pedagógicas e outros escritos (2000); Pedagogia da esperança: um reencontro com a pedagogia do oprimido (1992); La Educación como práctica de la Libertad (1997); Educação e mudança (s.f); Conscientização (1979); Ação cultural para a liberdade e outros escritos (1981); A importância do ato de ler (1989). 
En esta misma perspectiva, los aportes de Paulo Freire a la educación popular en el contexto de la Pedagogía Social Latinoamericana son básicamente tres: en primer lugar, el rol protagónico del educando, contrarrestando los efectos de la denominada "educación bancaria"6, en segundo lugar, la investigación participativa (ó IAP) argumentando que la educación es un proceso de praxis-reflexión-acción que el ser humano ejerce sobre el mundo para transformarlo con un claro propósito emancipador; y finalmente, la concepción metodológico-dialéctica que orienta el proceso global de transformación social, incluyendo la práctica educativa. Estos aportes constituyen el proceso del cual resulta la "teorización de la práctica", es decir la construcción de conceptos operativos que sintetizan los niveles de análisis e interpretación (Barreras, A.C. 2013).

El discurso pedagógico, filosófico y educativo que más se identifica con la Pedagogía Social en Latinoamérica es, sin lugar a dudas, el generado por la obra más importante de Paulo Freire, "Pedagogía del Oprimido" (1972), la cual se puede sintetizar en cuatro partes así: Una primera que esboza las razones de la Pedagogía del Oprimido, donde describe la contradicción opresores-oprimidos, con principios como nadie libera a nadie, nadie se libera solo; la segunda parte hace referencia a la concepción bancaria de la educación, el pensamiento problematizador

6. La concepción bancaria de la educación, según Freire, considera al educando como depositario de los contenidos ofrecidos por el educador, convirtiéndose en instrumento de opresión, pasividad, donde la memorización y la repetición son fin y un medio. El educador se considera poseedor de verdades únicas e inamovibles. de la educación y los argumentos del inacabamiento del ser humano; la tercera parte alude a la dialogicidad como esencia de la educación como práctica de la libertad, el poder del diálogo y las relaciones del hombre con el mundo; finalmente, la cuarta parte está referida a la dialogicidad y el antagonismo opresión-liberación a partir de los planteamientos teóricos acción dialógica y acción antidialógica. En esta obra Paulo Freire propone que el individuo se forme, no formarlo, para lo cual las experiencias de aprendizaje se deben generar en las vivencias de la cotidianidad y no de experiencias artificiales; dichos aprendizajes deben propender por la liberación de los sujetos.

La influencia del paradigma pedagógico de Paulo Freire ha sido de gran importancia en todos los niveles de los sistemas educativos, a partir del cual se han creado fundaciones, instituciones, centros educativos a su nombre; de otra parte se desarrollan cátedras universitarias, eventos, encuentros, seminarios y congresos, desde diversas miradas, se comparten, discuten y socializan experiencias, estudios e investigaciones generados a partir de su obra. Es relevante mencionar, por ejemplo, "el Primer Congreso Freireano Internacional Virtual de Educación Trasformadora para la Liberación" celebrado en agosto de 2011, en el que se resaltan temáticas como: "Siete miradas de Paulo Freire sobre educación" que según Mejía, M. R. (2011) describen diferentes perspectivas ancladas en áreas o disciplinas como:

1) Lo social, el transformador es transformado, desde dentro y desde fuera. 
2) Lo educativo, el educador necesita del educando y viceversa, ambos se educan.

3) Lo filosófico, sólo es posible dar nombre a las cosas, después que se hacen las cosas.

4) Lo psicológico, en el proceso del conocer es indispensable incluir la pasión, los sentimientos y el deseo.

5) Lo antropológico, el punto de partida de la educación está en el contexto cultural, ideológico, político y social de los educandos, no importa cómo está este contexto.

6) Lo espiritual, no se puede enseñar a amar, es preciso amar, el amor es la transformación definitiva.

7) Lo artístico, el educador debe ser sensible, esteta, tener gusto. La educación es una obra de arte, por tanto el educador es también un artista.

Complementando esta breve alusión a los desarrollos teóricos de la Pedagogía Social en América Latina, hacia la segunda mitad del siglo XX, es Orlando Fals Borda, el sociólogo y educador colombiano quien aportó ideas sociológicas, políticas y educativas, a través de su propuesta metodológica sobre Investigación Acción Participativa (IAP) ${ }^{7}$, la que ha generado influencia determinante en los investigadores sociales y educativos, con pautas orientadoras para cambio social en Colombia. Según Ocampo, J. (2009), Fals Borda fue fundador de la Facultad

7. El autor Ocampo López, Javier concluye que la investigación-acción participativa se define como un método de estudio y acción que va al paso con una filosofía altruista de la vida para obtener resultados útiles y confiables en el mejoramiento de situaciones colectivas, sobre todo para las clases populares. de Sociología en la Universidad Nacional de Colombia (1959), la primera en Latinoamérica, con el propósito central de formar sociólogos investigadores de la realidad social del país. Sus principales obras fueron "Campesinos de los Andes" (1955), "La violencia en Colombia, estudio de un proceso social" (1962), "El hombre y la tierra en Boyacá" (1957), "Revoluciones inconclusas en América Latina" (1968), "Historia de la cuestión agraria en Colombia" (1975), "Conocimiento y poder popular" (1980), "El reto del cambio social" (1985), "Ante la crisis del país, ideas-acción para el cambio" (2003), entre otras.

En el ámbito social, Fals Borda se interesó por promover el cambio social en Colombia a partir de investigaciones sobre movimientos campesinos, estudiantiles y sindicales; sus investigaciones hacen énfasis en los cambios socioculturales y la dinámica social en el contexto rural, como lo señala Ocampo, J. (2009, p. 20):

En los mediados del siglo XX, la mayor parte de las gentes vivían en los campos, dedicados a la agricultura y aún no había el problema de la emigración de los campesinos a las ciudades, como ocurrió en la segunda mitad del siglo XX.

No sobra reconocer que el aporte más significativo de Fals Borda es la aplicación en sus investigaciones del método de IAP, con el propósito de lograr cambios radicales en la sociedad a partir del compromiso y la conciencia de cambio; desde la perspectiva pedagógica el método busca generar acciones de solución a problemas reales y concretos, haciendo notar que hacer investigación a través de la IAP "es 
aceptar que toda investigación sea interacción comunicante, en la que ocurre un proceso de diálogo, de aprendizaje mutuo y de mutua confianza entre el investigador y el investigado" (Ocampo, J. 2009, p. 32).

Los diferentes análisis sobre el pensamiento de Orlando Fals Borda aluden al estudio del significado de la propuesta metodológica de la IAP, sobre esto último Souza, J.F. (2007) señala que la IAP "es una forma de organizar una praxis pedagógica $^{8}$ inspirada, o que toma sus bases en una Pedagogía o Teoría de la Educación, que en América Latina hemos denominado Educación Popular" (p. 15); en consecuencia, según Souza, la praxis pedagógica no es la misma práctica docente o práctica pedagógica (que hace referencia a la acción de los profesores o educadores), sino que es un concepto más amplio, supone una Pedagogía que ayude a las instituciones a formar nuevos profesionales o "seres humanos que se desempeñen humanamente" (p. 19). Esta concepción de praxis pedagógica se constituye en elemento generador de acciones institucionales en colectivo, organizadas formalmente en contextos culturales específicos para conseguir propósitos determinados. Esta concepción desafía el paradigma dominante y cataloga el pensamiento de Fals Borda como críti-

8. La praxis pedagógica es una categoría de análisis utilizada por Joao Francisco de Souza (2007) que significa la interrelación de prácticas de sujetos sociales que desean ser educados, respondiendo a los requerimientos de una determinada sociedad en un momento determinado de su historia; es decir, la praxis pedagógica, "es la acción institucional de la agencia formadora de cualquier formación, inclusive de la formación de profesores, la cual es responsable de una sociedad, o de sectores de esa sociedad" (p. 19). co-humanístico-emancipatorio y transformador, características propias de la educación popular en América Latina. En consecuencia, la expresión "praxis pedagógica" acuñada por Fals Borda, es más amplia que el concepto de "práctica pedagógica", referido solamente a la formación y acción del profesor que resulta suficiente para la comprensión, análisis y reinvención del quehacer de los maestros.

En el contexto de los análisis y reflexiones sobre la obra de Fals Borda, Souza, J.F. (2007, p. 24), concluye que la IAP "es un proceso que combina la investigación científica con la acción política, cuya meta es transformar radicalmente la realidad social y construir el poder popular". Es en esta perspectiva que la praxis pedagógica cobra sentido, en la medida en que la IAP, como instrumento, permite y contribuye a producir conocimiento adecuado para el cambio social y "consolidar nuevas relaciones sociales". El mismo autor describe seis premisas desde las cuales se fundamenta teóricamente la IAP: La razón práctica, como generadora de argumentos para intervenir en la realidad; conjunción del saber académico con el popular, que posibilita el diálogo de saberes académicos; relación sujeto/sujeto en la construcción de conocimiento; quiebre de la asimetría o compromiso existencial; epistemología holística o extensa, en contra de la reglas de trabajo positivistas-funcionalistas y; la interacción comunicante, aceptando que toda IAP es un proceso de diálogo de aprendizaje mutuo, de mutua confianza entre el investigador y el investigado (Souza, J.F. 2007, pp. 25-27). 


\section{Conclusión}

En los últimos años, el cambio de contexto del "sistema-mundo" descrito por Immanuel Wallerstein (1999) ${ }^{9}$, en los aspectos económico, político y sociocultural, han contribuido significativamente al auge y consolidación de la Pedagogía/Educación Social ${ }^{10}$, como alternativa teórico-práctica para los amplios ámbitos comunitarios de los marginados y desprotegidos que día a día aumentan en los países más pobres del mundo, incluyendo los latinoamericanos.

¿Cuáles serían las razones para consolidar, en Latinoamérica y particularmente en Colombia una Pedagogía/ Educación Social bajo fundamentos y principios que permitan generar líneas

9. WALLERSTEIN, Immanuel (1930) es un sociólogo y científico social histórico estadounidense interesado siempre por el análisis del sistema-mundo; sus obras se centran en una fuerte crítica al sistema capitalista mundial, haciendo parte del movimiento antiglobalización, junto con Noam Chomsky y Pierre Bourdieu, igualmente establece empatía intelectual con Ilya Prigogine (1917) y Frantz Fanon (1925) exponentes del desarrollo global de las ciencias sociales.

10. Para Gloria Pérez Serrano (2005) existe una distinción entre Pedagogía Social y Educación Social. Esta autora afirma: "Aunque en ocasiones, se utilizan indistintamente los términos Pedagogía Social y Educación Social, conviene indicar que la Pedagogía Social es una ciencia y que la Educación Social constituye su ámbito de intervención" (p. 10). Así, la pedagogía Social implica tanto el conocimiento como la acción y también la técnica o tecnología necesaria para la Educación Social de los seres humanos en condiciones normales o conflictivas. La misma autora agrega que la Pedagogía Social se la entiende como la ciencia de la Educación Social que centra su atención en el estudio de cuestiones inherentes a la socialización de los individuos y a la inadaptación de los mismos y orientada a la mejora de la calidad de vida.

Por su parte Kriekemans (1968) citado por Pérez (2005, p. 11), al referirse al objeto de la Educación social, afirma que ésta se propone "Formar el sentido social"; es decir promover entre los seres humanos unas buenas relaciones, donde el individuo se integra a su medio procurando desarrollar una conciencia adecuada y un sentido cívico. y proyectos de investigación, conformando una red de investigadores que dinamice y lidere desde la academia las orientaciones para la interacción/ acción en las comunidades marginadas, deprimidas y desplazadas?

Existen razones determinantes para fundamentar la constitución de una Pedagogía Social en Colombia, entre ellas la globalización, la crisis educativa, la violencia, la pobreza, el analfabetismo y el desplazamiento forzado, este último como un fenómeno creciente que se ha agudizado en los últimos años. Sin embargo éstos no son los únicos indicadores, que a la luz de las tendencias mundiales justifican la intencionalidad de instaurar de manera institucionalizada la Pedagogía Social en Colombia, como alternativa al desbordante incremento de necesidades educativas y sociales, especialmente en niños(as) y jóvenes. 


\section{BIBLIOGRAFÍA}

Adorno, T. (1998). Educación para la Emancipación. Madrid: Ediciones Morata.

Antón Valero, J.A. (2003). “La Pedagogía Crítica desde la Perspectiva de los Movimientos Sociales". En: Tabanque, 17, 51-70.

Añaños, F. (2006). (Comp.) Educación Social, Formación, Realidades y Retos. Granada: Universidad de Granada, Facultad de Educación. Colección Educación Social.

Añaños, F. (2010). La Integración Educativa y Sociocultural de los Inmigrantes en España, Realidades e Ideales. En Revista La Redvista, 1, 160-185.

Arias, R.L. (2006). Educación Social y formación ciudadana en tiempos de globalización. En Revista Tendencias y Retos, 11, 117-129.

Barreras, A. C. (2013). Las Tres Aportaciones, Paulo Freire. Disponible en: http://www.slideshare.net/ar. Fecha de consulta: 10-05-2013.

Boisvert, J. (2004). La Formación del Pensamiento Crítico. Teoría y Práctica. México: Editorial Fondo de Cultura Económica.

Caride, J. A. (2005). Las Fronteras de la Pedagogía Social, Perspectiva científica e histórica. Barcelona: Editorial Gedisa, S.A.

Carmona, G. (2010). Fundamentos Teórico-Epistemológicos de la Educación Social. Granada: Universidad de Granada.

Castro Lee, C. (2005). En Torno a la Violencia en Colombia, Una Propuesta Interdisciplinaria. Cali: Universidad del Valle.

Fals Borda, O. (1989). El Problema de cómo Investigar la realidad para Transformarla por la Praxis. Bogotá:Tercer mundo editores.

(1963). La Violencia en Colombia. Estudio de un Proceso Social.

(1986) Investigación Participativa.

(1998). Participación Popular.

Fanon, F. (1963). Los Condenados de la Tierra. México D.F.: Fondo de Cultura Económica, en Internet: http:// jokapala.myweb.uga.edu/violencia.html.

Fazio Vengoa, H. (2001). Globalización: discursos, imaginarios y realidades. Bogotá D.C.: Universidad de los Andes-IEPRI.

Fermoso, P. (1991). Teoría de la Educación. Madrid: Herder.

(1994). Pedagogía Social, Fundamentación Científica. Barcelona: Herder.

Fernández, A. \& Carmona, G. (2009). Re-hacer la Educación: los mapas del desarrollo humano. Teoría de la Educación, 47-56.

Fernández, M. y García, J (2010). Educación y Adultos Mayores. Rosario, Argentina: Laborde Libros Editor.

Freire, P. (1973). Extensión o Comunicación. Bogotá: Editorial América Latina.

Freire, P. (1976). Educación como Práctica de la Libertad. Bogotá: Editorial América Latina.

Freire, P. (1982). Pedagogía del Oprimido. Bogotá: Editorial América Latina.

Freire, P. (2008). La Importancia de Leer y el proceso de liberación. Madrid: Siglo XXI Editores.

Freire, P. (2009). Cartas a quien pretende enseñar. México: Siglo XXI Editores.

Freire, P. (2009). Pedagogía de la Esperanza. Madrid: Siglo XXI Editores. 
Freire, P. (s. f.). Cambio: El Rol del Trabajador Social en el Trabajo de Cambio. Bogotá: Editorial América Latina Freire, P. (2008).

García Mínguez, J. (2010).“Escenarios de la Pedagogía/Educación Social”. Documento de trabajo. Universidad de Granada, Facultad de Educación.

García Mínguez, J. (2010). Espacios de la Educación Social en Tiempos de Cambio. En Fernández, M.C. y García, J. (Comp.) Educación y Adultos Mayores. Rosario, Argentina: Universidad de Granada, Universidad Nacional de Rosario.

Gutiérrez, F. (1985). Educación como Praxis Política. México: Siglo XXI Editores.

Krichesky, M. (comp.). (2011). Pedagogía Social y Educación Popular: Perspectivas y Estrategias sobre la inclusión y el derecho a la educación. Buenos Aires, Universidad Pedagógica, UNIPE. Cuadernos de Trabajo No. 2.

Malagón, L. A. (2010). Las Ideas Pedagógicas de Paulo Freire. Bogotá D.C.: Editorial Magisterio.

Max-Neef, M., Elizande, A. y Hopenhayn, M. (1986). Desarrollo a Escala Humana, una opción para el futuro. Santiago: Cepaur, Fundación Dag Hammarskjold.

Mejía, M.R. (2001). Pedagogía en la Educación Popular, Reconstruyendo una opción político- pedagógica en la globalización. (Ponencia). Bogotá: Expedición Pedagógica Nacional - Fe y Alegría.

Mejía, M. R. (2005). “Vigencia Educativa de Freire”, en Revista Aportes, 58, 59-68.

Mejía, M. R. (2011). Primer Congreso Freiriano Internacional Virtual de Educación Transformadora para la Liberación. Disponible en http://peruforopaulofreire.blogspot.com. Fecha de consulta: 12-05- 2013.

Nájera, E. M. (2011). A la búsqueda del sujeto pedagógico. Encuentros entre pedagogía social y educación popular en Chile. En Krichesky, M. (comp.). Pedagogía Social y Educación Popular. Perspectivas y estrategias sobre la inclusión y el derecho a la educación (37- 50). Buenos Aires: Universidad Pedagógica, UNIPE. Cuadernos de Trabajo No. 2.

Narváez, L. C. (2002): La Pobreza en América Latina y particularmente en Colombia, Málaga: Universidad de Málaga. En internet: http://www.eumed.net/cursecon/ecolat/co/lcnt-pobreza. Fecha de consulta: 13 octubre de 2010.

Núñez, V. (2002). La Educación en Tiempos de Incertidumbre, Las apuestas de la Pedagogía Social. Barcelona: Editorial Gedisa.

Ocampo, J. (2009). “El Maestro Orlando Fals Borda, sus Ideas Educativas y Sociales para el Cambio en la Sociedad Colombiana". En Revista Historia de la Educación Latinoamericana, Rhela. Vol. 12, 13-41.

Ojeda, F. (2006). Sociedad Multicultural: Educación Intercultural. En Añaños, F. (Comp.). Educación Social, Formación, Realidad y Retos (133-141). Granada, Grupo Editorial Universitario.

Organización de las Naciones Unidas para la Educación, la Ciencia y la Cultura, Unesco (2010). Informe de Seguimiento de la EPT en el Mundo, “Llegar a los Marginados". París: Ediciones Unesco.

Romao, J. E. (2008). “Globalización y Reforma de la Educación en Brasil”. Brasilia D.F.

Souza, J. F. (2007). La contribución del pensamiento de Orlando Fals Borda a la teoría de la Educación. En J. Souza, D. Santos, C. Vasco, M. Quintero, C. Echavarría, E. Vasco (Eds). Investigación Acción en Educación en Contextos de Pobreza, un Homenaje a la Vida y Obra del Maestro Orlando Fals Borda. Bogotá D.C.: Universidad de la Salle.

Touraine, A. (2009). La Mirada Social. Un marco de pensamiento distinto para el siglo XXI. Barcelona: Ediciones Paidós Ibérica, S.A.

Triana, A. N. (2012). Educación Social y la Educación Popular en América Latina. Módulo de Pedagogía Social. Tunja: Universidad Pedagógica y Tecnológica de Colombia

Úcar, X. (2011). De Europa a Latinoamérica: hacia una Perspectiva Global, Compleja e Integrada de la Pedagogía Social. Barcelona: Departamento de Pedagogía Sistemática y Social, Universidad de Barcelona.

Zemelman, H. (2007). Epistemología de la Conciencia Histórica. México: Instituto Pensamiento y Cultura en América Latina. IPECAL. 